\title{
Optical conductivity of granular aluminum films near the Mott metal-to-insulator transition
}

\author{
Aviv Glezer Moshe, ${ }^{1,2}$ Eli Farber, ${ }^{2}$ and Guy Deutscher ${ }^{1}$ \\ ${ }^{1}$ Raymond and Beverly Sackler School of Physics and Astronomy, Tel Aviv University, Tel Aviv, Israel \\ ${ }^{2}$ Department of Physics and Department of Electrical and Electronic Engineering, Ariel University, P.O.B. 3, Ariel 40700, Israel.
}

(Dated: June 5, 2019)

\begin{abstract}
We report measurements of the energy gap of granular aluminum films by THz spectroscopy. We find that as the grains progressively decouple, the coupling ratio $2 \Delta(0) / k_{B} T_{c}$ increases above the BCS weak coupling ratio 3.53 , and reaches values consistent with an approach to BCS-BEC crossover for the high resistivity samples, expected from the short coherence length. The Mattis-Bardeen theory describes remarkably well the behavior of $\sigma_{1, s} / \sigma_{1, n}$ for all samples up to very high normal state resistivities.
\end{abstract}

\section{INTRODUCTION}

In granular superconductors, electrons are confined inside nano-scale metallic grains due to inter-grain oxide barriers, which reduces the value of the coherence length. When the barrier thickness is increased, the coherence volume can decrease up to a point where the number of Cooper pairs that it contains is of order unity. Thereby, a cross-over from a BCS regime where the number of pairs per coherence volume is very large to a BEC condensation where it is of order unity is expected. However, a detailed comparison with experiments was so far difficult because of a lack of theoretical studies of the BCS to BEC crossover regime. But recently, detailed theoretical predictions regarding the evolution of the strong coupling ratio around this crossover have become available [1, 2]. We show here that they are in quantitative agreement with our experimental findings, obtained on granular aluminum films from $\mathrm{THz}$ optical conductivity measurements in the vicinity of the metal to insulator transition.

We find that up to that transition the optical gap edge remains well defined and that, contrary to the behavior of disordered NbN films [3], the ratio $2 \Delta(0) / k_{B} T_{c}$ increases with resistivity, reaching a value of 4.51 in the highest resistivity sample $(\sim 8,000 \mu \Omega \mathrm{cm})$ studied.

According to the recent work of Pisani et al. [1, 2], the strong coupling ratio increases when the BCS to BCE crossover is approached. The highest resistivity sample that we have studied falls in the range where the strong coupling ratio is substantially enhanced. It is consistent with the experimental value of the coherence length.

In view of their possible applications as high kinetic inductance elements in quantum circuits (QC), there has been recently renewed interest in the properties of strongly disordered superconductors in the vicinity of the metal to insulator (M/I) transition. The optical properties of disordered $\mathrm{NbN}$ films have been the focus of particular attention [3, 4]. It was found that in these films, where disorder has a homogeneous distribution on the atomic scale, the coupling ratio $2 \Delta(0) / k_{B} T_{c}$ decreases continuously below the weak coupling limit value 3.53 when disorder is increased. This decrease becomes very pronounced when the parameter $k_{F} l$, where $k_{F}$ is the Fermi wave vector and $l$ the electron mean free path, becomes of order unity [3]. At the highest resistivity investigated $(\sim 1,000 \mu \Omega \mathrm{cm})$ there is not even a clear optical gap edge. At the same time the coupling ratio obtained from

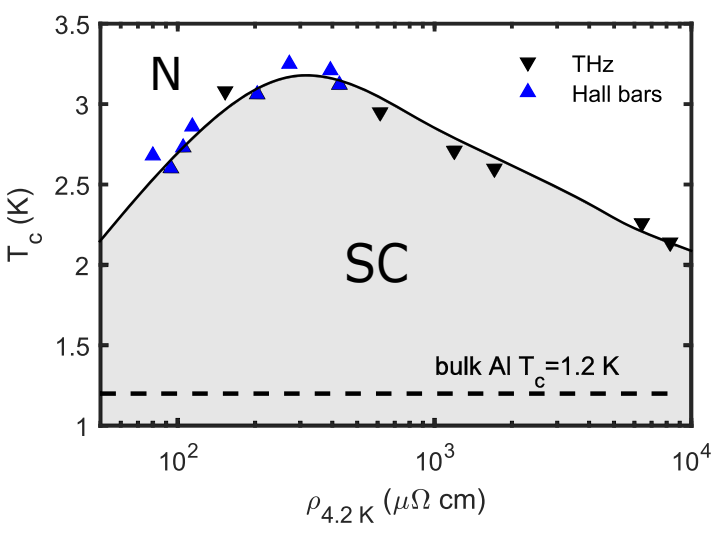

Figure 1. Critical temperature of studied granular aluminum films versus the normal state resistivity. Black upside down triangles $(\boldsymbol{\nabla})$ marks $T_{c}$ of the optical spectroscopy studied samples. Blue triangles ( $\boldsymbol{\Delta}$ ) marks $T_{c}$ as measured by Hall bars with similar deposition conditions as described in the main text. The dashed line marks the bulk aluminum critical temperature.

the tunneling gap remains at about 4.2. This behavior departs from the predictions of the BCS Mattis-Bardeen theory [5]. The enhanced low frequency dissipation has detrimental consequences on the quality factor of high impedance resonators that could be used in QC [6].

The behavior of granular $\mathrm{Al}$ is more favorable for $\mathrm{QC}$ applications. We propose that the difference in behavior between $\mathrm{NbN}$ and granular $\mathrm{Al}$ films stems from the different nature of the metal to insulator transition, being of the Anderson type in the former [7] and of the Mott type in the latter [8].

\section{EXPERIMENTAL AND METHODS}

Granular aluminum thin films were prepared by thermal evaporation of clean al pallets in a controlled $\mathrm{O}_{2}$ pressure, where the base pressure of the vacuum chamber is $\sim 1 \times 10^{-7}$ Torr. The films were deposited onto liquid nitrogen cooled substrates $\left(10 \times 10 \times 2 \mathrm{~mm}^{3} \mathrm{MgO}\right.$ or $10 \times 10 \times 1 \mathrm{~mm}^{3}$ LSAT, which result in similar fabry-perot pattern in the optical spectra). Films with various degree of grain coupling 


\begin{tabular}{cccccc}
\hline \hline Sample ID & $\rho_{300 K}(\mu \Omega \mathrm{cm})$ & $\rho_{4.2 K}^{n}(\mu \Omega \mathrm{cm})$ & $k_{F} l$ & $T_{c}(\mathrm{~K})$ & $\frac{2 \Delta(0)}{k_{B} T_{c}}$ \\
\hline 1 & 155 & 153 & 4.38 & 3.08 & 3.56 \\
2 & 589 & 614 & 1.00 & 2.95 & 3.64 \\
3 & 1047 & 1193 & 0.55 & 2.71 & 3.73 \\
4 & 1482 & 1710 & 0.36 & 2.60 & 3.77 \\
5 & 5131 & 6415 & 0.10 & 2.26 & 4.29 \\
6 & 6344 & 8265 & 0.085 & 2.14 & 4.51 \\
\hline \hline
\end{tabular}

Table I. Studied samples properties.

were obtained by varying the $\mathrm{O}_{2}$ partial pressure in the range of $2-5 \times 10^{-5}$ Torr while keeping the deposition rate about $5 \pm 1 \mathrm{~A} / \mathrm{s}$, similar to previous work [9, 10]. The films thickness varied in the range $40-100 \mathrm{~nm}$ in order to obtain high quality transmission measurements as the resistivity of the films increases.

Standard four point resistivity measurements were performed in either a commercial QD PPMS or in a home built probe, we characterize each film by its normal state resistivity $\rho_{n}$ at $4.2 \mathrm{~K}$. Great care was taken to obtain homogeneous films. Sharp superconducting transitions were obtained even for the highest resistivity films, see Fig. 3 .

The optical spectroscopy measurements were done by utilizing a quasi-optical Mach-Zehnder interferometer which allows us to obtain the complex transmission $\hat{t}=|t| e^{i \phi_{t}}$ [9, 11] of the substrate-film system. The radiation sources are tunable monochromatic backward-wave oscillators (BWO), by utilizing several sources we cover a frequency range of $3-17 \mathrm{~cm}^{-1}$ (or about $0.1-0.5 \mathrm{THz}$ ). Commercial optical ${ }^{4} \mathrm{He}$ cryostat with a home built sample holder, provides us dynamic temperature range down to $1.5 \mathrm{~K}$ and the ability to measure up to two samples during one cooldown. The complex transmission was measured for all samples at $4.2 \mathrm{~K}$ and at various temperatures close to and below $T_{c}$ down to $1.5 \mathrm{~K}$. Then the complex conductivity $\hat{\sigma}(\omega)=\sigma_{1}(\omega)+i \sigma_{2}(\omega)$ is calculated from the measured complex transmission via the Fresnel equations [11, 12], without relying on any microscopic model.

\section{RESULTS}

Fig. 1 shows the superconducting (SC) critical temperature as a function of the normal state resistivity at $4.2 \mathrm{~K}$ for films studied in this work and also that of Hall bars prepared separately in our lab. The well known "dome" shaped phase diagram [9, 13, 15] can be clearly seen.

To allow an easy comparison with the behavior of $\mathrm{NbN}$ films [3, 4] our main results are displayed Table I. To evaluate $k_{F} l$ we approximate it by the free electron formula $k_{F} l=$ $\frac{m}{\hbar} \frac{1}{\rho} v_{F} \rho l$ by using the values of $v_{F}=15.99 \times 10^{7} \mathrm{~cm} / \mathrm{s}$ and $\rho l=5.01 \times 10^{-6} \mu \Omega \mathrm{cm}^{2}$ as obtained by Gall [16] for clean aluminum. For all samples, except for sample 1, the value of $k_{F} l$ is equal to or smaller than one, in sharp contrast with the behavior of the studied NbN films [3, 4].

The normal state conductivity $\sigma_{1, n}$ as measured at $4.2 \mathrm{~K}$

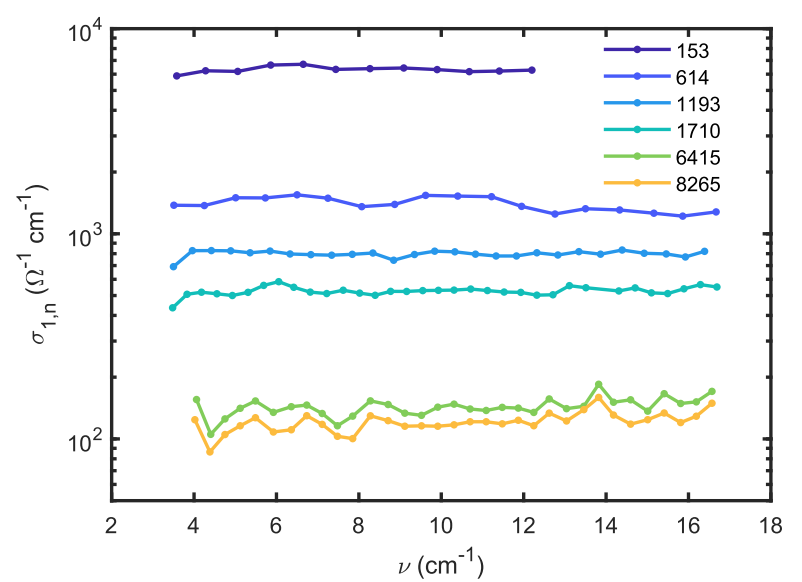

Figure 2. Real part of the optical conductivity at $4.2 \mathrm{~K}$ for all samples. The legend marks the normal state resistivity in $\mu \Omega \mathrm{cm}$ as obtained from transport measurements of the same samples.

is frequency independent up to $17 \mathrm{~cm}^{-1}$, see Fig. 2. The resistive SC transition for all studied samples, along with their measured SC gap $\Delta(T)$ as a function of reduced temperature $T / T_{c}$ is presented in Fig. 3, where we define $T_{c}$ as the temperature where the resistivity has decreased below $1 \%$ of its normal state value. Up to the highest resistivity samples the superconducting transition is well defined, see Fig. 4.

In the superconducting state a good fit to the MB theory [5] is obtained for all samples up to resistivities of about 8,000 $\mu \Omega \mathrm{cm}$, as one can see in Fig. 5. Values of $\Delta(0)$ used in table I were obtained from these fits.

In figures 6, 5 and 2 the frequency $\nu$ is given in units of $\mathrm{cm}^{-1}$ which is related to $\omega$ by $\omega=2 \pi c \nu$, where $c$ is the speed of light in $\mathrm{cm} / \mathrm{sec}$. We analyze the optical data similarly to the method used by Pracht et al. [9]. Representative transmission $|t|^{2}$ and frequency normalized phase spectra $\phi / \nu$ are shown in Fig. 6, for both normal (at $4.2 \mathrm{~K}$ ) and superconducting state. The strong oscillation pattern is due to multiple reflections inside the substrate. Utilizing the Fresnel equations for multiple reflections in a bilayer system [11, 12, 17], we obtain the complex conductivity $\hat{\sigma}(\omega)=\sigma_{1}(\omega)+i \sigma_{2}(\omega)$, without relying on any microscopic model. The measured transmission depends on both the substrate and film dielectric function $\hat{\epsilon}(\omega)$ and thickness

$$
\hat{t}=t\left(d_{s}, \epsilon_{1}^{s}(\omega), \epsilon_{2}^{s}(\omega) ; d_{f}, \epsilon_{1}^{f}(\omega), \epsilon_{2}^{f}(\omega)\right)
$$

where $\hat{\epsilon}(\omega)=\epsilon_{1}(\omega)+i \epsilon_{2}(\omega)$ which can be expressed as complex conductivity $\hat{\sigma}(\omega)$ by the relation

$$
\hat{\epsilon}(\omega)=1+i \frac{2 \pi}{\epsilon_{0}} \frac{\hat{\sigma}(\omega)}{\omega}
$$

Once $\hat{t}$ is measured, we need to disentangle the substrate contribution in order to extract the complex conductivity of 

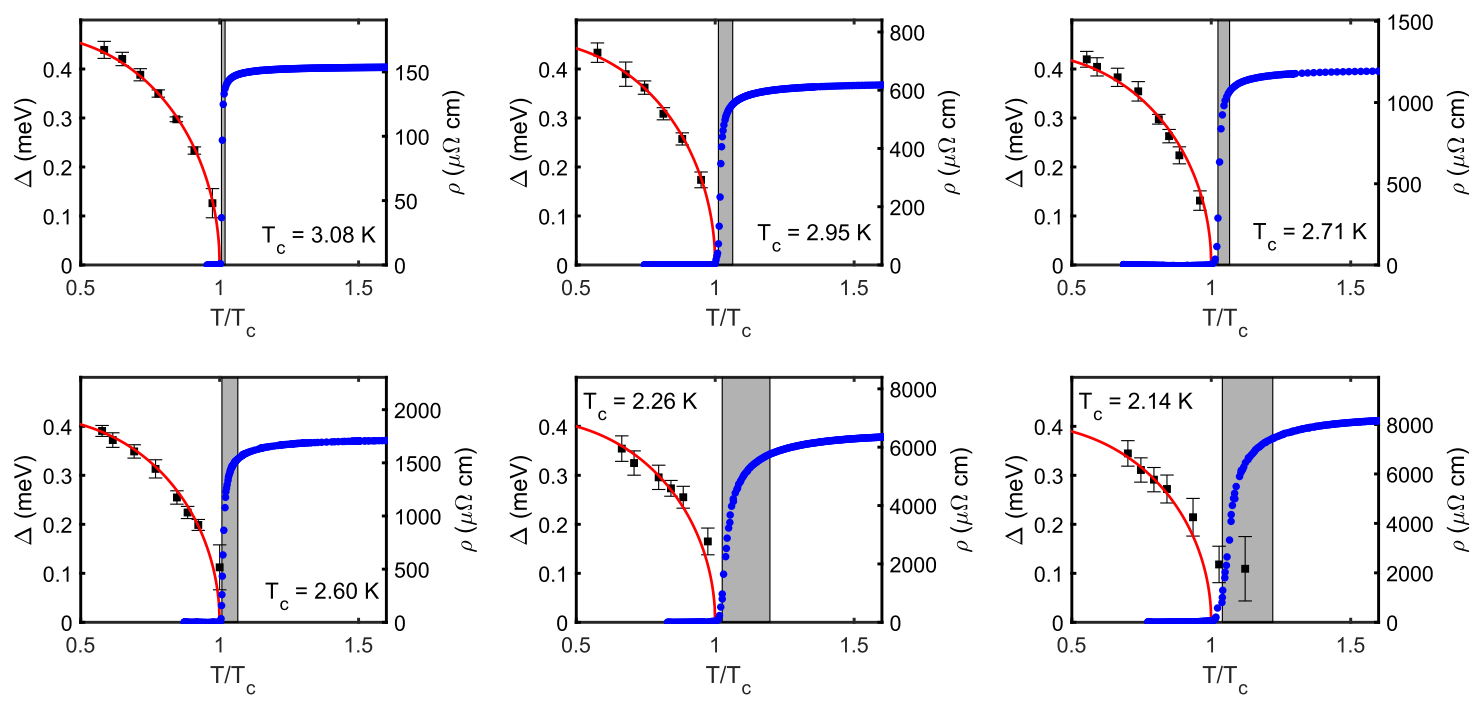

Figure 3. $\Delta(T)$ and $\rho(T)$ versus temperature for all studied samples. Black squares ( $)$ are the measured gap, obtained by fitting $\sigma_{1, s} / \sigma_{1, n}$ to MB formulae and the red curve is a fit to a BCS gap equation curve. Blue circles $(\bullet)$ are the measured resistivity and the grey area marks the decrease of the normal state resistivity from 90 to $10 \%$ of its normal state value.

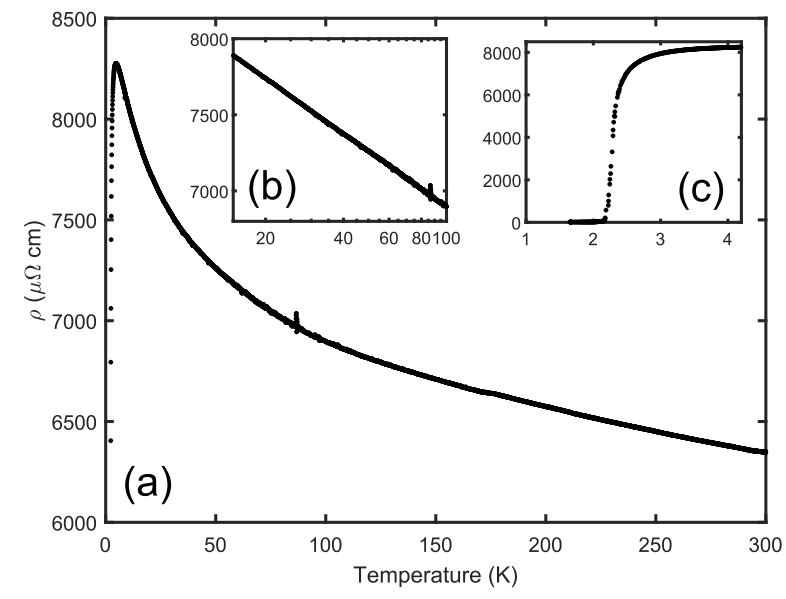

Figure 4. (a) Resistivity as a function of temperature of sample 6. Insets (b-c) are the same data focused on different temperatures scales: (b) The temperature is presented in a logarithmic scale, which shows logarithmic increase of $\rho$ over wide range of temperatures. (c) The resistive $\mathrm{SC}$ transition.

the film. The $\mathrm{MgO}$ and LSAT dielectric substrates are known to be completely transparent (means $\epsilon_{2}^{s}=0$ ) in the THz frequency range [9, 18], as we also checked in a separate experiment. We first analyze the normal state at $4.2 \mathrm{~K}$, where the normal state conductivity obeys Drude model with high scattering rate relatively to the measured frequency range, in this low frequency regime $\sigma_{2} \simeq 0$. We fit around each max$\mathrm{ima} /$ minima in the spectra with $\sigma_{1}$ of the film and $\epsilon_{1}$ of the substrate. We then use the obtained set of $\epsilon_{1}$ for the low temperature data where we fit around each maxima/minima by both $\sigma_{1}, \sigma_{2}$ of the film. This process yields a pair of $\sigma_{1}, \sigma_{2}$ for each frequency point, as can be shown in Fig. 6

Once we obtain $\hat{\sigma}(\omega, T)$ we fit it to the Mattis-Bardeen (MB) formulas [5], which are appropriate since we are well in the dirty limit $\Delta \ll \hbar / \tau$ [9, 10, 19]

$$
\begin{aligned}
\frac{\sigma_{1}(\omega)}{\sigma_{n}}= & \frac{e^{2} n_{s}}{m^{*} \sigma_{n}} \pi \delta(\omega) \\
& +\frac{2}{\hbar \omega} \int_{\Delta}^{\infty} d E g(E)[f(E)-f(E+\hbar \omega)] \\
& -\frac{\Theta(\hbar \omega-2 \Delta)}{\hbar \omega} \int_{\Delta-\hbar \omega}^{-\Delta} d E g(E)[1-2 f(E+\hbar \omega)] \\
\frac{\sigma_{2}(\omega)}{\sigma_{n}}= & \frac{1}{\hbar \omega} \int_{\max \{-\Delta, \Delta-\hbar \omega\}}^{\Delta} d E g(E)[1-2 f(E+\hbar \omega)] \\
& \times \frac{E(E+\hbar \omega)+\Delta^{2}}{\sqrt{\Delta^{2}-E^{2}} \sqrt{(E+\hbar \omega)^{2}-\Delta^{2}}} \\
& g(E)=\frac{E(E+\hbar \omega)+\Delta^{2}}{\sqrt{E^{2}-\Delta^{2}} \sqrt{(E+\hbar \omega)^{2}-\Delta^{2}}}
\end{aligned}
$$

The normal state conductivity is determined as $\sigma_{1}(\omega)$ at $4.2 \mathrm{~K}$, we then fit $\sigma_{1} / \sigma_{n}$ with $\Delta$ as the sole fitting parameter. For some of the samples the behavior of $\sigma_{1}(\omega)$ at subgap frequencies $\omega<2 \Delta / \hbar$ slightly deviates from MB and therefore are being excluded from the fit. We then fit $\Delta(T)$ to the standard BCS gap equation [20] with $\Delta(0)$ as the sole fitting parameter, without any restriction on the coupling ratio $2 \Delta(0) / k_{B} T_{c}$. The complex conductivity for all samples at the lowest measured temperature along with its MB fit is shown in Fig. 5 with good agreement for all samples. 

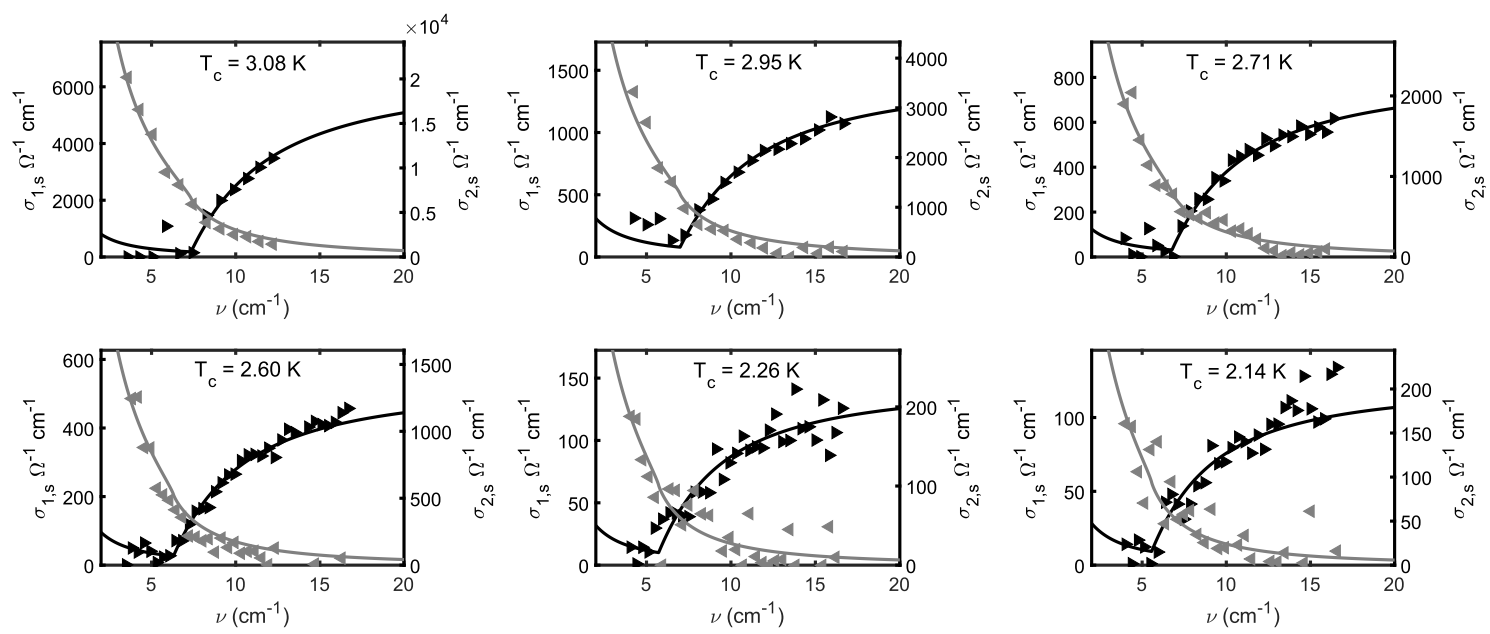

Figure 5. Optical conductivity $\hat{\sigma}(\omega)$ of all samples. The black triangles and line are the real part of the conductivity and its MB fit, the grey triangles and line are the imaginary part of the conductivity and its MB fit. The data is shown for $T=1.5 \mathrm{~K}$, except for sample 2 $\left(T_{c}=2.95 \mathrm{~K}\right)$ which was measured down to $T=1.7 \mathrm{~K}$.

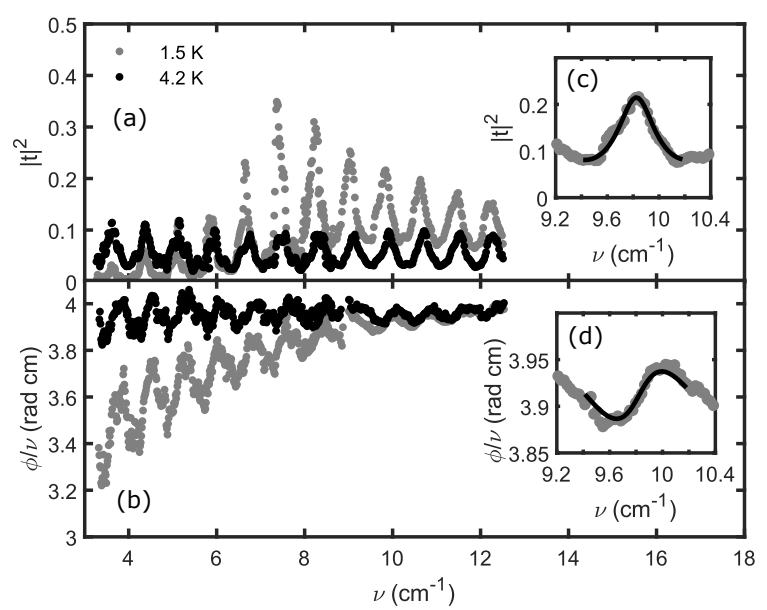

Figure 6. Raw data of sample 1. (a) Transmittance. (b) phase divided by frequency in the normal (black) and SC (grey) state. Insets (c) and (d) show an example of the resulting simultaneous fit to both $|t|^{2}$ and $\phi_{t} / \nu$ (black line) by $\sigma_{1}, \sigma_{2}$ of the film.

\section{DISCUSSION}

First of all we compare our results to tunneling data. The value of the optical gap as determined from fitting the conductivity data to the MB theory is close to the value of the tunneling gap reported by Abeles and Hanak [21]. According to them, the coupling ratio $2 \Delta(0) / k_{B} T_{c}$ is equal to 3.4 for all specimen within $2 \%$. In fact, according to the optical gap values reported in Table. 1, the coupling ratio increases continuously with resistivity, a trend already noted by Pracht et al. [9] for a high resistivity sample. This deviation appears to be systematic, the coupling ratio varying from 3.56 for the lowest resistivity sample (close to the weak coupling value of

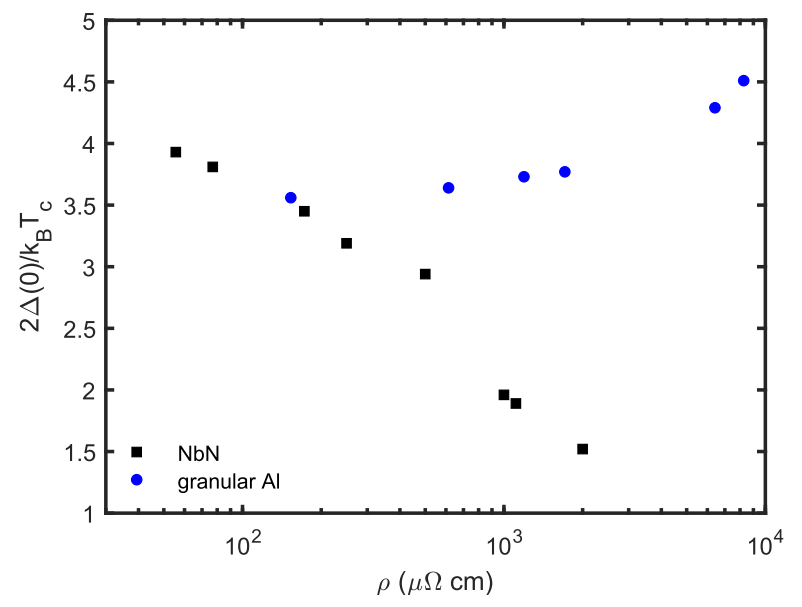

Figure 7. Coupling ratio versus resistivity. Black squares are $\mathrm{NbN}$ data from Ref. [3] and blue circles are our granular Al data.

3.53) up to 4.51 for the highest resistivity one. This is one of our main findings.

Second of all, we compare our results with those obtained by Cheng et al. on disordered $\mathrm{NbN}$ films [3]. As can be seen in Fig. 7. as the resistivity (and $\left(k_{F} l\right)^{-1}$ ) increases, the coupling ratio decreases continuously, rather than increasing. Furthermore, the value of the optical gap becomes distinct from and smaller than the tunneling gap. At the highest resistivity investigated $(\sim 1,000 \mu \Omega \mathrm{cm})$ there is not even a clear optical gap edge. This difference in behavior between the optical data obtained on granular $\mathrm{Al}$ and $\mathrm{NbN}$ is emphasized in Fig. 8 , where $\hbar \omega$ has been scaled by twice the value of the tunneling gap. While for granular Al the conductivities scale fairly, they clearly don't for $\mathrm{NbN}$.

These points deserve a detailed discussion.

Regarding the first one, the increase of the coupling ratio 

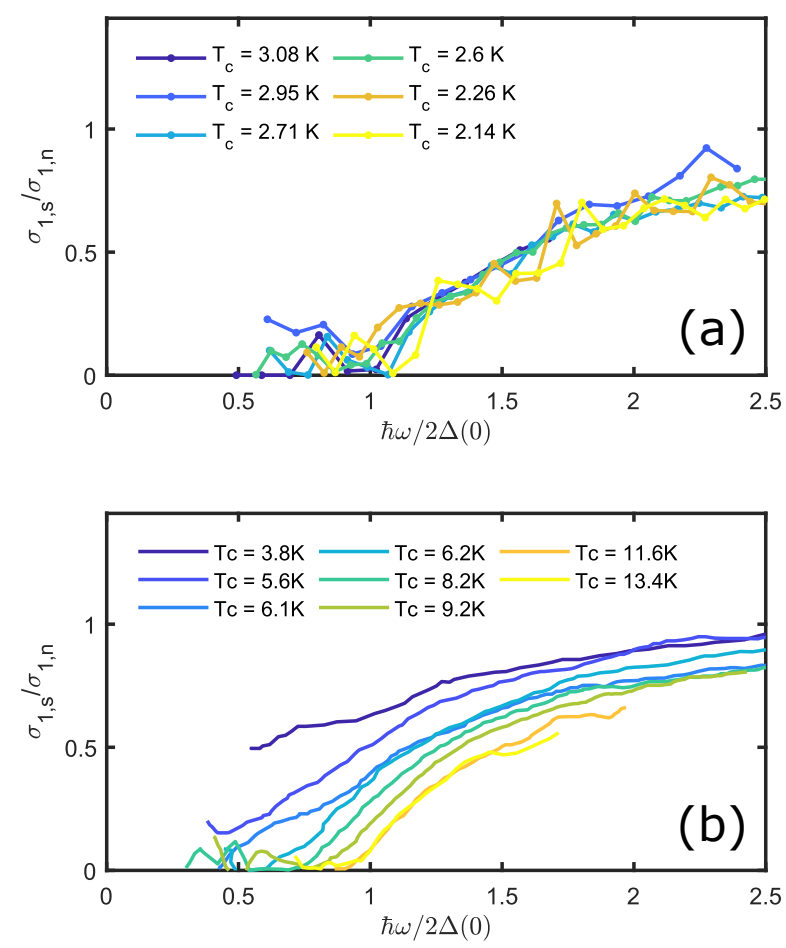

Figure 8. Real part of the optical conductivity, measured at the lowest temperature, normalized to the normal state conductivity. The frequency axis is normalized to twice the value of the tunneling gap. (a) our granular $\mathrm{Al}$ data (b) Cheng et al. $\mathrm{NbN}$ data [3].

seen in granular Al with resistivity may have two different origins. The first one would be a stronger coupling to soft phonons often cited as the possible reason for the higher $T_{c}$ of granular films [22]. However, we discard this interpretation here since the critical temperature is decreasing in our series of high resistivity samples. A second origin would be an approach to a BCS to BEC crossover. In the case of a $\mathrm{BE}$ condensation, the pair breaking energy becomes disconnected from the $k_{B} T_{c}$ energy scale, which is much smaller. In the crossover regime from BCS to BE condensation the coupling ratio becomes progressively larger than the weak coupling limit value. This effect has been studied recently in detail by Pisani et al. [1, 2] who have calculated how $T_{c} / E_{F}$, the zero temperature gap $\Delta(0) / E_{F}$ and $\frac{2 \Delta}{k_{B} T_{c}}$ vary with the distance to the unitary limit, where the product of the Fermi wave vector $k_{F}$ by the scattering length $a_{F}$ goes to infinity. We note that it is possible to describe the crossover in terms of the variable $k_{F} \xi_{\text {pair }}$ [23], which has been calculated as a function of $\left(k_{F} a_{F}\right)^{-1}$ [24].

We favor this second interpretation. The idea is that in the granular case confinement results in strong coupling in the sense that the coherence length can become so short that there are only a few pairs in a coherence volume, which is what happens at a BCS to BEC cross-over. When the coupling ratio reaches the value of 4.51 , as is the case for our highest

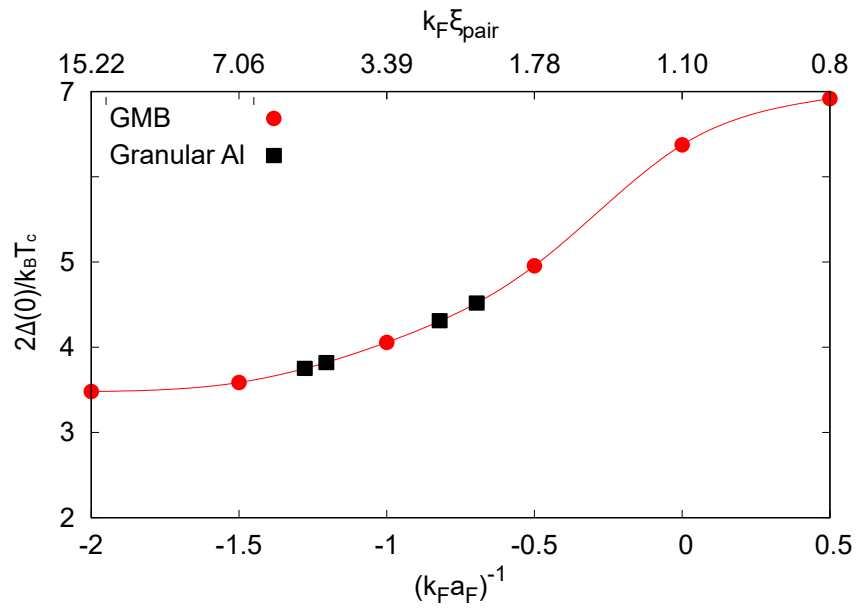

Figure 9. The coupling ratio as a function of $k_{F} \xi_{\text {pair }}$ or $\left(k_{F} a_{F}\right)^{-1}$ (Pisani et al., private communication). The red circles $(\bullet)$ are the results of the numerical calculations that include corrections beyond mean field which arise from pairing fluctuations, labeled GMB. The red line joining these circles is a guide to the eye. The black squares (ם) correspond to our measured coupling ratio. The corresponding values of $\left(k_{F} a_{F}\right)^{-1}$ range from -1.3 to -0.7 and those of $k_{F} \xi_{\text {pair }}$ from 5.3 to 2.3 .

resistivity sample, $\left(k_{F} a_{F}\right)^{-1} \simeq-0.70$,or $k_{F} \xi_{\text {pair }} \simeq 2.28$ according to Pistolesi et al. [23], not far from the unitary limit. Fig. 9 shows the coupling ratios of samples 3-6, for samples 1,2 the coupling ratio is insensitive to variation in $\left(k_{F} a_{F}\right)^{-1}$ and therefore is not shown. We use the measured coupling ratio to evaluate $E_{F}$ and therefore $k_{F}$ (assuming that the effective mass is not too different than that of a bare electron). For our most resistive sample $\Delta(0) / E_{F}=0.17$ which yields $E_{F}=2.47 \mathrm{meV}$ and $k_{F}=2.54 \times 10^{8} \mathrm{~m}^{-1}$. Upper critical field measurements of similar samples [25] yield a coherence length of about $\xi_{0} \simeq 10 \mathrm{~nm} \simeq \xi_{\text {pair }}$. We obtain $k_{F} \xi_{\text {pair }}=2.54$, which is close to the value obtained by the coupling ratio alone. A reduced effective Fermi energy was previously inferred from the negative magneto- resistance seen in granular Al films. It was analyzed in terms of the presence of diluted magnetic impurities [8], but this model does not apply when $k_{F} l$ is smaller than unity, as is the case here. This may be the reason why the reduction in $E_{F}$ was not as strong as seen here.

Concerning the second point, one can understand the difference in behavior between granular $\mathrm{Al}$ and disordered $\mathrm{NbN}$ films as resulting from their different disorder length scales, being respectively the grain size in granular $\mathrm{Al}$ and the lattice parameter or inter-atomic distance in $\mathrm{NbN}$. In a 3D system the metal to insulator transition occurs when the conductivity $g$ measured at the relevant length scale crosses a critical value $g_{c}$. Here $g$ is the universal conductance $\frac{2 e^{2}}{h}$ divided by the relevant length scale $d$. Since the grain size in granular $\mathrm{Al}$ is about one order of magnitude larger than the interatomic distance in $\mathrm{NbN}$, it follows that the value of the critical conductivity in the former is expected to be about one order of magnitude smaller than in the latter. However this argument does not explain the qualitative difference between the behav- 
iors of the optical conductivities of granular $\mathrm{Al}$ and $\mathrm{NbN}$ seen in Fig. 8. As the M/I transition is approached a sharp gap edge persists in granular $\mathrm{Al}$ but not in $\mathrm{NbN}$. This suggests that the nature of the $\mathrm{M} / \mathrm{I}$ transition is not the same in the two systems. While it is of the Anderson type in NbN, we believe that it is of the Mott type in granular Al, as already proposed [8]. This is consistent with the large value of the coupling ratio, since at a Mott transition the effective bandwidth reduces to zero (while the DOS at the Fermi level remains finite [26]). As the bandwidth reduces the coupling ratio increases for any finite value of the gap. In DMFT models the ratio of the Coulomb energy to the bandwidth is close to 3 when the transition occurs [26]. In the granular case the value of the Coulomb energy is that of the electrostatic energy of the grains, which for a $2 \mathrm{~nm}$ size grain is about $30 \mathrm{meV}$ [27]. An effective bandwidth of 10 $\mathrm{meV}$ is compatible with the value of $2.5 \mathrm{meV}$ for the Fermi energy deduced from the value of the strong coupling ratio. We note that the energy level splitting is also about $10 \mathrm{meV}$.

We may add that the coherence length in $\mathrm{NbN}$ is always much larger than the disorder length scale, so that the system never approaches the BCS to BEC cross-over and therefore never develops the strong coupling effect predicted by Pisani et al. [1, 2]. In fact in $\mathrm{NbN}$ the coherence length increases when the metal to insulator transition is approached [28]. It is worthy to note that Cao et al. [29] showed that in a superlattice made from 'magic angle' twisted bilayer graphene (TBG) superconductivity appears as the TBG it is doped slightly away from the Mott-like insulator state. In the superconducting state, a proximity to BCS-BEC crossover is possible, supported by $\xi \approx 50 \mathrm{~nm}$ and inter-particle spacing of about $26 \mathrm{~nm}$.

\section{CONCLUSION}

Optical spectroscopy reveals that a sharp gap edge persists in granular Aluminum films as the metallic grains are being decoupled towards the Metal to Insulator transition. Remarkably, at the same time the coupling ratio $2 \Delta(0) / k_{B} T_{c}$ increases, reaching in the most resistive sample a value consistent with an approach to the unitary limit. The critical temperature is not strongly reduced by the grain decoupling. This behavior is consistent with a Mott transition driven by the electrostatic charging energy of the grains.

This behavior is in contrast with that observed previously in atomically disordered $\mathrm{NbN}$ film, where the coupling ratio reduces when disorder is increased, the critical temperature goes down strongly and the sharp gap edge vanishes, a behavior consistent with an Anderson transition [7]. The origin of the good performance of high kinetic inductance granular $\mathrm{Al}$ resonators reported recently [6] may be due to the fact that the $\mathrm{M} / \mathrm{I}$ transition is of the Mott type rather than of the Anderson type. This granular system may indeed be viewed as a network of Josephson junctions between well-defined $\mathrm{Al}$ grains as suggested by Grünhaupt et al. [6], rather than as a highly disordered superconductor.

\section{ACKNOWLEDGMENTS}

We acknowledge G. C. Strinati for fruitful discussion and for supplying us with Fig. 9 data. We are grateful to N. Bachar for guidance with early sample preparation and $\mathrm{THz}$ measurements.

*avivmoshe@mail.tau.ac.il
[1] L. Pisani, P. Pieri, and G. C. Strinati, Phys. Rev. B 98, 104507 (2018)

[2] L. Pisani, A. Perali, P. Pieri, and G. C. Strinati, Phys. Rev. B 97, 014528 (2018)

[3] B. Cheng, L. Wu, N. J. Laurita, H. Singh, M. Chand, P. Raychaudhuri, and N. P. Armitage, Phys. Rev. B 93, 180511 (2016)

[4] D. Sherman, U. S. Pracht, B. Gorshunov, S. Poran, J. Jesudasan, M. Chand, P. Raychaudhuri, M. Swanson, N. Trivedi, A. Auerbach, M. Scheffler, A. Frydman, and M. Dressel, $\mathrm{Na}-$ ture Physics 11, 188 (2015)

[5] D. C. Mattis and J. Bardeen, Phys. Rev. 111, 412 (1958).

[6] L. Grünhaupt, N. Maleeva, S. T. Skacel, M. Calvo, F. LevyBertrand, A. V. Ustinov, H. Rotzinger, A. Monfardini, G. Catelani, and I. M. Pop, Phys. Rev. Lett. 121, 117001 (2018).

[7] M. Mondal, A. Kamlapure, M. Chand, G. Saraswat, S. Kumar, J. Jesudasan, L. Benfatto, V. Tripathi, and P. Raychaudhuri, Phys. Rev. Lett. 106, 047001 (2011).

[8] N. Bachar, S. Lerer, A. Levy, S. Hacohen-Gourgy, B. Almog, H. Saadaoui, Z. Salman, E. Morenzoni, and G. Deutscher, Physical Review B 91, 041123 (2015).

[9] U. S. Pracht, N. Bachar, L. Benfatto, G. Deutscher, E. Farber, M. Dressel, and M. Scheffler, Physical Review B 93, 100503
(2016)

[10] N. Bachar, U. Pracht, E. Farber, M. Dressel, G. Deutscher, and M. Scheffler, Journal of Low Temperature Physics 179, 83 (2015)

[11] U. S. Pracht, E. Heintze, C. Clauss, D. Hafner, R. Bek, D. Werner, S. Gelhorn, M. Scheffler, M. Dressel, D. Sherman, B. Gorshunov, K. S. Il'in, D. Henrich, and M. Siegel, IEEE Transactions on Terahertz Science and Technology 3, 269 (2013)

[12] M. Dressel and G. Gruner, eds., Cambridge University Press, 2002).

[13] N. Bachar, S. Lerer, S. Hacohen-Gourgy, B. Almog, and G. Deutscher, Physical Review B 87, 214512 (2013)

[14] G. Deutscher, M. Gershenson, E. Grunbaum, and Y. Imry, Journal of Vacuum Science and Technology 10, 697 (1973)

[15] G. Deutscher, H. Fenichel, M. Gershenson, E. G. nbaum, and Z. Ovadyahu, Journal of Low Temperature Physics 10, 231 (1973)

[16] D. Gall, Journal of Applied Physics 119, 085101 (2016). https://doi.org/10.1063/1.4942216.

[17] B. Gorshunov, A. Volkov, I. Spektor, A. Prokhorov, A. Mukhin, M. Dressel, S. Uchida, and A. Loidl, International Journal of Infrared and Millimeter Waves 26, 1217 (2005) 
[18] S. Arezoomandan, A. Prakash, A. Chanana, J. Yue, J. Mao, S. Blair, A. Nahata, B. Jalan, and B. Sensale-Rodriguez, Scientific Reports 8, 3577 (2018)

[19] U. S. Pracht, T. Cea, N. Bachar, G. Deutscher, E. Farber, M. Dressel, M. Scheffler, C. Castellani, A. M. GarcÃa-GarcÃa, and L. Benfatto, Physical Review B 96, 094514 (2017)

[20] J. Bardeen, L. N. Cooper, and J. R. Schrieffer, Physical Review 108, 1175 (1957)

[21] B. Abeles and J. Hanak, Physics Letters A 34, 165 (1971).

[22] J. W. Garland, K. H. Bennemann, and F. M. Mueller, Phys. Rev. Lett. 21, 1315 (1968)

[23] F. Pistolesi and G. C. Strinati, Phys. Rev. B 49, 6356 (1994)

[24] G. C. Strinati, P. Pieri, G. Ropke, P. Schuck, and M. Urban,
Physics Reports 738, 1 (2018), the BCS - BEC crossover: From ultra-cold Fermi gases to nuclear systems.

[25] S. Lerer, N. Bachar, G. Deutscher, and Y. Dagan, Physical Review B 90, 214521 (2014)

[26] A. Georges, G. Kotliar, W. Krauth, and M. J. Rozenberg, Rev. Mod. Phys. 68, 13 (1996)

[27] B. Abeles, Physical Review B 15, 2828 (1977)

[28] M. Chand, G. Saraswat, A. Kamlapure, M. Mondal, S. Kumar, J. Jesudasan, V. Bagwe, L. Benfatto, V. Tripathi, and P. Raychaudhuri, Phys. Rev. B 85, 014508 (2012)

[29] Y. Cao, V. Fatemi, S. Fang, K. Watanabe, T. Taniguchi, E. Kaxiras, and P. Jarillo-Herrero, Nature 556, 43 (2018) 